

\title{
Chai's conjecture and Fubini properties of dimensional motivic integration
}

\author{
Raf Cluckers, François Loeser and Johannes Nicaise
}

\begin{abstract}
We prove that a conjecture of Chai on the additivity of the base change conductor for semiabelian varieties over a discretely valued field is equivalent to a Fubini property for the dimensions of certain motivic integrals. We prove this Fubini property when the valued field has characteristic zero.
\end{abstract}

\section{Introduction}

Let $R$ be a henselian discrete valuation ring with quotient field $K$ and perfect residue field $k$. Let $G$ be a semiabelian variety over $K$, i.e., an extension of an abelian $K$-variety by a $K$-torus. Then $G$ can be canonically extended to a smooth separated commutative group scheme $\mathscr{G}$ over $R$, the so-called Néron lft-model of $G$ [Bosch et al. 1990, 10.1.1]. We say that $G$ has semiabelian reduction if the identity component of the special fiber of $\varphi$ is a semiabelian $k$-variety.

Chai [2000] introduced the base change conductor $c(G)$ of $G$, a positive rational number that measures the defect of semiabelian reduction of $G$. Its precise definition is recalled in Definition 2.3.1. The base change conductor vanishes if and only if $G$ has semiabelian reduction. For algebraic tori, this invariant had previously been defined and studied by Chai and Yu [2001]. They proved the deep result that the base change conductor of a $K$-torus $T$ is invariant under isogeny. Applying an argument from [Gross and Gan 1999], they deduced that $c(T)$ equals one half of the Artin conductor of the cocharacter module of $T$. For semiabelian varieties, however, no similar cohomological interpretation is known to hold in general; in fact, the base change conductor is not even invariant under isogeny [Chai 2000, §6.10], and many of its properties remain mysterious. One of the main open questions is the following conjecture:

The authors received funding from the European Research Council (Grant Agreement 246903 NMNAG) under the European Community's Seventh Framework Programme and from the Fund for Scientific Research - Flanders (G.0415.10).

MSC2010: primary 14K15; secondary 03C65, 03C98, 11G10.

Keywords: semiabelian varieties, motivic integration, base change conductor. 
Conjecture 1.1 [Chai 2000, §8.1]. Let $G$ be a semiabelian $K$-variety that fits into an exact sequence of algebraic $K$-groups

$$
0 \rightarrow T \rightarrow G \rightarrow A \rightarrow 0
$$

with $T$ a $K$-torus and $A$ an abelian $K$-variety. Then we have

$$
c(G)=c(A)+c(T) .
$$

The fundamental difficulty underlying this conjecture is that an exact sequence of semiabelian varieties does not give rise to an exact sequence of Néron lft-models in general. Chai proved the conjecture if $k$ is finite, using Fubini's theorem for integrals with respect to the Haar measure on the completion of $K$. He also proved the conjecture when $K$ has mixed characteristic, using a different method and applying the property that $c(T)$ only depends on the isogeny class of $T$. If $k$ has characteristic zero (more generally, if $G$ obtains semiabelian reduction after a tame finite extension of $K$ ), Chai's conjecture can be proven in an elementary way; see [Halle and Nicaise 2011, 4.23].

In the first part of the present paper, we show that, in arbitrary characteristic, Chai's conjecture is equivalent to a Fubini property for the dimensions of certain motivic integrals (Equation (4.2-1) in Theorem 4.2.1). We then prove in the second part of the paper that this Fubini property holds when $K$ has characteristic zero (Theorem 4.2.3). This yields a new proof of the conjecture in that case, which is close in spirit to Chai's proof of the finite residue field case.

The strength of our approach lies in the fact that we combine insights of two theories of motivic integration, namely, the geometric theory of motivic integration on rigid varieties of Loeser and Sebag [2003] and the model-theoretic approach of Cluckers and Loeser [2008; 2012]. Let us emphasize that the Fubini property in (4.2-1) is not an immediate corollary of the Fubini results from [Cluckers and Loeser 2012]; see Remark 4.2.5. We need to combine the theory in [Cluckers and Loeser 2012] with a new result (Theorem 5.2.1 and its corollary), which roughly states that the virtual dimension of a motivic integral over a fixed space only depends on the dimensions of the values of the integrand. This theorem may be of independent interest.

We hope that our reformulation of Chai's conjecture in terms of motivic integrals will also shed new light on the open case of the conjecture, when $k$ is infinite, $K$ has positive characteristic and $G$ is wildly ramified.

\section{Preliminaries}

2.1. Notation. Throughout this article, $R$ denotes a henselian discrete valuation ring with quotient field $K$ and perfect residue field $k$. We denote by $\mathfrak{m}$ the maximal 
ideal of $R, R^{s h}$ a strict henselization of $R$ and $K^{s h}$ its field of fractions. The residue field $k^{s}$ of $R^{s h}$ is an algebraic closure of $k$. We denote by $\widehat{R}$ the $\mathfrak{m}$-adic completion of $R$ and $\widehat{K}$ its field of fractions.

For every ring $A$, we denote by (Sch/A) the category of $A$-schemes. We consider the special fiber functor

$$
(\cdot)_{k}:(\mathrm{Sch} / R) \rightarrow(\mathrm{Sch} / k): \mathscr{X} \mapsto \mathscr{X}_{k}=X \times_{R} k
$$

and the generic fiber functor

$$
(\cdot)_{K}:(\mathrm{Sch} / R) \rightarrow(\mathrm{Sch} / K): \mathscr{X} \mapsto \mathscr{X}_{K}=\mathscr{X} \times_{R} K .
$$

A variety over a $\operatorname{ring} A$ is a reduced separated $A$-scheme of finite type.

2.2. Néron models and semiabelian reduction. A semiabelian variety over a field $F$ is an extension of an abelian $F$-variety by an algebraic $F$-torus. Let $G$ be a semiabelian variety over $K$. It follows from [Bosch et al. 1990, 10.2.2] that $G$ admits a Néron lft-model $\mathscr{G}$ in the sense of [Bosch et al. 1990, 10.1.1]. It is the minimal extension of $G$ to a smooth separated group scheme over $R$. We say that $G$ has semiabelian reduction if the identity component $\varphi_{k}^{o}$ of the special fiber of $G$ is a semiabelian $k$-variety. There always exists a finite separable extension $L$ of $K$ such that $G \times{ }_{K} L$ has semiabelian reduction. If $G$ is an abelian variety, then this is Grothendieck's semistable reduction theorem [Grothendieck et al. 1972, IX.3.6]. If $G$ is a torus, then one can take for $L$ the splitting field of $G$. The general case is easily deduced from these special cases; see [Halle and Nicaise 2010, 3.11].

Let $K^{\prime}$ be a finite separable extension of $K$, and denote by $R^{\prime}$ the integral closure of $R$ in $K^{\prime}$. We set $G^{\prime}=G \times{ }_{K} K^{\prime}$, and we denote by $\varphi^{\prime}$ the Néron lft-model of $G^{\prime}$. By the universal property of the Néron lft-model, there exists a unique morphism of $R^{\prime}$-schemes

$$
h: \mathscr{G}_{R} R^{\prime} \rightarrow \mathscr{G}^{\prime}
$$

that extends the natural isomorphism between the generic fibers. If $G$ has semiabelian reduction, then $h$ is an open immersion [Grothendieck et al. 1972, 3.1(e)], which induces an isomorphism

$$
\left(\mathscr{G} \times{ }_{R} R^{\prime}\right)^{o} \rightarrow\left(\varphi^{\prime}\right)^{o}
$$

between the identity components of $\varphi_{{ }^{\prime}}{ }_{R} R^{\prime}$ and $\varphi^{\prime}$ [Demazure and Grothendieck 1970a, $\left.\mathrm{VI}_{\mathrm{B}} \cdot 3.11\right]$.

2.3. The base change conductor. Let $G$ be a semiabelian variety over $K$. Let $K^{\prime}$ be a finite separable extension of $K$ such that $G^{\prime}=G \times{ }_{K} K^{\prime}$ has semiabelian reduction, and denote by $e\left(K^{\prime} / K\right)$ the ramification index of $K^{\prime}$ over $K$. The 
morphism (2.2-1) induces an injective morphism

$$
\operatorname{Lie}(h): \operatorname{Lie}(\mathscr{G}) \otimes_{R} R^{\prime} \rightarrow \operatorname{Lie}\left(\varphi^{\prime}\right)
$$

of free $R^{\prime}$-modules of $\operatorname{rank} \operatorname{dim}(G)$.

Definition 2.3.1 [Chai 2000, §1]. The base change conductor of $G$ is defined by

$$
c(G)=\frac{1}{e\left(K^{\prime} / K\right)} \cdot \text { length }_{R^{\prime}}(\operatorname{coker}(\operatorname{Lie}(h))) .
$$

This definition does not depend on the choice of $K^{\prime}$. The base change conductor is a positive rational number that vanishes if and only if $G$ has semiabelian reduction [Halle and Nicaise 2011, 4.16]. One can view $c(G)$ as a measure for the defect of semiabelian reduction of $G$.

2.4. A generalization of Chai's conjecture. Chai [2000, 8.1] asks whether one can generalize Conjecture 1.1 as follows.

Question 2.4.1. Do we have $c\left(G_{2}\right)=c\left(G_{1}\right)+c\left(G_{3}\right)$ for every exact sequence of semiabelian $K$-varieties

$$
0 \rightarrow G_{1} \rightarrow G_{2} \rightarrow G_{3} \rightarrow 0 ?
$$

If $G_{1}, G_{2}$ and $G_{3}$ are tori, this can be easily deduced from the deep fact that the base change conductor of a torus is one half of the Artin conductor of the cocharacter module [Chai and Yu 2001] in the following way:

Proposition 2.4.2. Let

$$
0 \rightarrow G_{1} \rightarrow G_{2} \rightarrow G_{3} \rightarrow 0
$$

be an exact sequence of $K$-tori. Then $c\left(G_{2}\right)=c\left(G_{1}\right)+c\left(G_{3}\right)$.

Proof. The sequence of cocharacter modules

$$
0 \rightarrow X_{\bullet}\left(G_{1}\right) \rightarrow X_{\bullet}\left(G_{2}\right) \rightarrow X_{\bullet}\left(G_{3}\right) \rightarrow 0
$$

is exact. Tensoring with $\mathbb{Q}$, we get a split exact sequence of $\mathbb{Q}[\mathrm{Gal}(L / K)]$-modules

$$
0 \rightarrow X_{\bullet}\left(G_{1}\right) \otimes_{\mathbb{Z}} \mathbb{Q} \rightarrow X_{\bullet}\left(G_{2}\right) \otimes_{\mathbb{Z}} \mathbb{Q} \rightarrow X_{\bullet}\left(G_{3}\right) \otimes_{\mathbb{Z}} \mathbb{Q} \rightarrow 0
$$

where $L$ is the splitting field of $G_{2}$. Thus, the Artin conductor of $X_{\bullet}\left(G_{2}\right) \otimes_{\mathbb{Z}} \mathbb{Q}$ is the sum of the Artin conductors of $X_{\bullet}\left(G_{1}\right) \otimes_{\mathbb{Z}} \mathbb{Q}$ and $X_{\bullet}\left(G_{3}\right) \otimes_{\mathbb{Z}} \mathbb{Q}$. Since the base change conductor of a torus is one half of the Artin conductor of the cocharacter module [Chai and Yu 2001], we find that $c\left(G_{2}\right)=c\left(G_{1}\right)+c\left(G_{3}\right)$.

Corollary 2.4.3. If Conjecture 1.1 holds, then Question 2.4.1 has a positive answer when $G_{1}$ is a torus. 
Proof. Assume that $G_{1}$ is a torus. For every semiabelian $K$-variety $G$, we denote by $G_{\text {tor }}$ its maximal subtorus and $G_{\mathrm{ab}}=G / G_{\text {tor }}$ its abelian part. We consider the closed subgroup $\widetilde{G}_{2}=\left(G_{3}\right)_{\text {tor }} \times_{G_{3}} G_{2}$ of $G_{2}$. We have a short exact sequence of $K$-groups

$$
0 \rightarrow G_{1} \rightarrow \widetilde{G}_{2} \rightarrow\left(G_{3}\right)_{\text {tor }} \rightarrow 0
$$

so that $\widetilde{G}_{2}$ is an extension of $K$-tori and thus a torus. Moreover, the morphism

$$
G_{2} / \widetilde{G}_{2} \rightarrow G_{3} /\left(G_{3}\right)_{\mathrm{tor}}=\left(G_{3}\right)_{\mathrm{ab}}
$$

is an isomorphism so that $\widetilde{G}_{2}=\left(G_{2}\right)_{\text {tor }}$ and $\left(G_{2}\right)_{\mathrm{ab}} \cong\left(G_{3}\right)_{\mathrm{ab}}$. By Conjecture 1.1, we have $c\left(G_{i}\right)=c\left(\left(G_{i}\right)_{\mathrm{tor}}\right)+c\left(\left(G_{i}\right)_{\mathrm{ab}}\right)$ for $i=2$, 3. Applying Proposition 2.4.2 to the sequence $(2.4-1)$, we find that $c\left(\left(G_{2}\right)_{\text {tor }}\right)=c\left(G_{1}\right)+c\left(\left(G_{3}\right)_{\text {tor }}\right)$. It follows that $c\left(G_{2}\right)=c\left(G_{1}\right)+c\left(G_{3}\right)$.

Below, we will follow a different approach. We will use the invariance of the base change conductor of a torus under isogeny to reduce Question 2.4.1 to the case where the maximal split subtorus $\left(G_{3}\right)_{\mathrm{sp}}$ of $G_{3}$ is trivial (of course, this is always the case if $G_{3}$ is an abelian variety as in Conjecture 1.1). Then we prove that, if $G_{1}$ is a torus and $\left(G_{3}\right)_{\mathrm{sp}}$ is trivial, the additivity property of the base change conductor in Question 2.4.1 is equivalent to a certain Fubini property for motivic integrals. We prove this Fubini property when $K$ has characteristic zero. These arguments do not use the invariance of the base change conductor of a torus under isogeny.

\section{Motivic Haar measures on semiabelian varieties}

3.1. The Grothendieck ring of varieties. Let $F$ be a field. We denote by $K_{0}\left(\operatorname{Var}_{F}\right)$ the Grothendieck ring of varieties over $F$. As an abelian group, $K_{0}\left(\operatorname{Var}_{F}\right)$ is defined by the following presentation:

generators: isomorphism classes $[X]$ of separated $F$-schemes of finite type $X$, relations: if $X$ is a separated $F$-scheme of finite type and $Y$ is a closed subscheme of $X$, then

$$
[X]=[Y]+[X \backslash Y] .
$$

These relations are called scissor relations.

By the scissor relations, one has $[X]=\left[X_{\text {red }}\right]$ for every separated $F$-scheme of finite type $X$, where $X_{\text {red }}$ denotes the maximal reduced closed subscheme of $X$. We endow the group $K_{0}\left(\operatorname{Var}_{F}\right)$ with the unique ring structure such that

$$
[X] \cdot\left[X^{\prime}\right]=\left[X \times_{F} X^{\prime}\right]
$$

for all separated $F$-schemes of finite type $X$ and $X^{\prime}$. The identity element for the multiplication is the class [Spec $F$ ] of the point. To any constructible subset $C$ of a 
separated $F$-scheme of finite type $X$, one can associate an element $[C]$ in $K_{0}\left(\operatorname{Var}_{F}\right)$ by choosing a finite partition of $C$ into subvarieties $C_{1}, \ldots, C_{r}$ of $X$ and setting $[C]=\left[C_{1}\right]+\cdots+\left[C_{r}\right]$. The scissor relations imply that this definition does not depend on the choice of the partition. For a detailed survey on the Grothendieck ring of varieties, we refer to [Nicaise and Sebag 2011a].

We denote by $K_{0}^{\bmod }\left(\operatorname{Var}_{F}\right)$ the modified Grothendieck ring of varieties over $F$ [Nicaise and Sebag 2011a, §3.8]. This is the quotient of $K_{0}\left(\operatorname{Var}_{F}\right)$ by the ideal $\rrbracket_{F}$ generated by elements of the form $[X]-[Y]$ where $X$ and $Y$ are separated $F$ schemes of finite type such that there exists a finite, surjective, purely inseparable $F$-morphism $Y \rightarrow X$. If $F$ has characteristic zero, then it is easily seen that $\Phi_{F}$ is the zero-ideal [Nicaise and Sebag 2011a, 3.11] so that $K_{0}\left(\operatorname{Var}_{F}\right)=K_{0}^{\bmod }\left(\operatorname{Var}_{F}\right)$. It is not known if $\mathscr{I}_{F}$ is nonzero if $F$ has positive characteristic. In particular, if $F^{\prime}$ is a nontrivial finite purely inseparable extension of $F$, it is not known whether $\left[\operatorname{Spec} F^{\prime}\right] \neq 1$ in $K_{0}\left(\operatorname{Var}_{F}\right)$.

There exists a canonical isomorphism from $K_{0}^{\bmod }\left(\operatorname{Var}_{F}\right)$ to the Grothendieck ring $K_{0}\left(\mathrm{ACF}_{F}\right)$ of the theory $\mathrm{ACF}_{F}$ of algebraically closed fields over $F$ [Nicaise and Sebag 2011a, 3.13]. One may also consider the semiring variant $K_{0}^{+}\left(\mathrm{ACF}_{F}\right)$ of the ring $K_{0}\left(\mathrm{ACF}_{F}\right)$, defined as follows. Let $\mathscr{L}_{\text {ring }}(F)$ be the ring language with coefficients from $F$. As a semigroup, $K_{0}^{+}\left(\mathrm{ACF}_{F}\right)$ is the quotient of the free commutative semigroup generated by a symbol $[X]$ for each $\mathscr{L}_{\text {ring }}(F)$-definable set, with zero-element $[\varnothing]$, and divided out by the following relations:

- if $X$ and $Y$ are $\mathscr{L}_{\text {ring }}(F)$-definable subsets of a common $\mathscr{L}_{\text {ring }}(F)$-definable set, then

$$
[X \cup Y]+[X \cap Y]=[X]+[Y]
$$

- if there exists an $\mathscr{L}_{\text {ring }}(F)$-definable bijection $X \rightarrow Y$ for the theory $\mathrm{ACF}_{F}$, then $[X]=[Y]$.

The semigroup $K_{0}^{+}\left(\mathrm{ACF}_{F}\right)$ carries a structure of semiring, induced by taking Cartesian products, $[X][Y]=[X \times Y]$.

If $R$ has equal characteristic, then we put

$$
K_{0}^{R}\left(\operatorname{Var}_{k}\right)=K_{0}\left(\operatorname{Var}_{k}\right)
$$

If $R$ has mixed characteristic, then we put

$$
K_{0}^{R}\left(\operatorname{Var}_{k}\right)=K_{0}^{\bmod }\left(\operatorname{Var}_{k}\right) .
$$

We denote by $\mathbb{L}$ the class of the affine line $\mathbb{A}_{k}^{1}$ in $K_{0}^{R}\left(\operatorname{Var}_{k}\right)$ and also in $K_{0}^{+}\left(\mathrm{ACF}_{k}\right)$. We will write $M_{k}^{R}$ for the localization of $K_{0}^{R}\left(\operatorname{Var}_{k}\right)$ with respect to $\mathbb{L}$ and $\mathcal{M}_{k}^{+}$for the localization of $K_{0}^{+}\left(\mathrm{ACF}_{k}\right)$ with respect to $\mathbb{L}$ and the elements $\mathbb{L}^{i}-1$ for all $i>0$. 
For every element $\alpha$ of $K_{0}^{R}\left(\operatorname{Var}_{k}\right)$, we denote by $P(\alpha)$ its Poincaré polynomial [Nicaise and Sebag 2011a, 4.13]. This is an element of $\mathbb{Z}[T]$, and the map

$$
P: K_{0}^{R}\left(\operatorname{Var}_{k}\right) \rightarrow \mathbb{Z}[T]: \alpha \mapsto P(\alpha)
$$

is a ring morphism. Hence, the map

$$
P^{+}: K_{0}^{+}\left(\mathrm{ACF}_{k}\right) \rightarrow \mathbb{Z}[T],
$$

obtained by composing $P$ with the canonical morphism

$$
K_{0}^{+}\left(\mathrm{ACF}_{F}\right) \rightarrow K_{0}\left(\mathrm{ACF}_{F}\right) \cong K_{0}^{R}\left(\operatorname{Var}_{k}\right),
$$

is a morphism of semirings. When $\alpha$ is the class of a separated $k$-scheme of finite type $X$, then for every $i \in \mathbb{N}$, the coefficient of $T^{i}$ in $P(\alpha)$ is $(-1)^{i}$ times the $i$-th virtual Betti number of $X$. The degree of $P(\alpha)$ is twice the dimension of $X$ [Nicaise 2011, 8.7].

We have $P(\mathbb{L})=T^{2}$ so that $P$ localizes through a ring morphism

$$
P: M_{k}^{R} \rightarrow \mathbb{Z}\left[T, T^{-1}\right]
$$

and $P^{+}$localizes through a semiring morphism

$$
P^{+}: \mathcal{M}_{k}^{+} \rightarrow \mathbb{Z}\left[T, T^{-1},\left(T^{2 i}-1\right)^{-1}\right]_{i>0} .
$$

Definition 3.1.1. Let $\alpha$ be an element of $\mathcal{M}_{k}^{R}$ or $\mathcal{M}_{k}^{+}$. We define the virtual dimension of $\alpha$ as $1 / 2$ times the degree of the Poincaré polynomial $P(\alpha)$ or $P^{+}(\alpha)$, respectively, where the degree of the zero-polynomial is $-\infty$ and $(1 / 2) \cdot(-\infty)=-\infty$ by convention. We denote the virtual dimension of $\alpha$ by $\operatorname{dim}(\alpha)$.

By definition, the virtual dimension is an element of $(1 / 2) \cdot \mathbb{Z} \cup\{-\infty\}$. For every separated $k$-scheme of finite type $X$ and every integer $i$, we have

$$
\operatorname{dim}\left([X] \mathbb{Q}^{i}\right)=\operatorname{dim}(X)+i .
$$

3.2. Motivic integration on $K$-varieties. Let $X$ be a $K$-variety. We say that $X$ is bounded if $X\left(K^{s h}\right)$ is bounded in $X$ in the sense of [Bosch et al. 1990, 1.1.2]. If $X$ is a smooth $K$-variety, then by [Bosch et al. 1990, 3.4.2 and 3.5.7], $X$ is bounded if and only if $X$ admits a weak Néron model $\mathscr{X}$. This means that $\mathscr{X}$ is a smooth $R$-variety endowed with an isomorphism $\mathscr{X}_{K} \rightarrow X$ such that the natural map

$$
\mathscr{L}\left(R^{s h}\right) \rightarrow X\left(K^{s h}\right)
$$

is a bijection.

The theory of motivic integration on rigid varieties was developed in [Loeser and Sebag 2003] and further extended in [Nicaise and Sebag 2008; Nicaise 2009]. We refer to [Nicaise and Sebag 2011b] for a survey; see in particular [Nicaise and Sebag 
$2011 \mathrm{~b}, \S 2.4]$ for an erratum to the previous papers. One of the main results can be reformulated for algebraic varieties as follows. Let $X$ be bounded smooth $K$-variety of pure dimension, and let $\omega$ be a gauge form on $X$, i.e., a nowhere-vanishing differential form of degree $\operatorname{dim}(X)$. Let $\mathscr{X}$ be a weak Néron model for $X$. For every connected component $C$ of $\mathscr{X}_{k}=\mathscr{X} \times_{R} k$, we denote by $\operatorname{ord}_{C} \omega$ the order of $\omega$ along $C$. If $\varpi$ is a uniformizer in $R$, then $\operatorname{ord}_{C} \omega$ is the unique integer $n$ such that $\varpi^{-n} \omega$ extends to a generator of $\Omega_{\mathscr{L} / R}^{\operatorname{dim}(X)}$ at the generic point of $C$.

Theorem-Definition 3.2.1. The object

$$
\int_{X}|\omega|=\mathbb{L}^{-\operatorname{dim}(X)} \sum_{C \in \varpi_{0}\left(\mathscr{X}_{k}\right)}[C] \mathbb{L}^{-\operatorname{ord}_{C} \omega} \in \mathcal{M}_{k}^{R}
$$

only depends on $X$ and $\omega$ and not on the choice of a weak Néron model $\mathscr{X}$. We call it the motivic integral of $\omega$ on $X$.

Proof. By [Nicaise 2011, 4.9], the formal $\mathfrak{m}$-adic completion of $\mathscr{X}$ is a formal weak Néron model of the rigid analytification $X^{\text {rig }}$ of $X \times_{K} \widehat{K}$ so that the result follows from [Halle and Nicaise 2011, 2.3].

It is clear from the definition that the motivic integral of $\omega$ on $X$ remains invariant if we multiply $\omega$ with a unit in $R$.

Remark 3.2.2. In the literature, the factor $\mathbb{L}^{-\operatorname{dim}(X)}$ in the right-hand side of (3.2-1) is sometimes omitted (for instance in [Nicaise and Sebag 2011b]); this depends on the choice of a normalization for the motivic measure.

3.3. Motivic Haar measures. Consider a semiabelian $K$-variety $G$ of dimension $g$. We denote by $G$ the Néron lft-model of $G$ and $\Omega_{G}$ the free rank-one $R$-module of translation-invariant differential forms in $\Omega_{\mathscr{G} / R}^{g}(\mathscr{G})$. Note that $\Omega_{G} \otimes_{R} K$ is canonically isomorphic to the $K$-vector space of translation-invariant differential forms of maximal degree on $G$ so that we can view $\Omega_{G}$ as an $R$-lattice in this vector space. We denote by $\omega_{G}$ a generator of $\Omega_{G}$. It is unique up to multiplication with a unit in $R$.

Let $K^{\prime}$ be a finite separable extension of $K$ such that $G^{\prime}=G \times{ }_{K} K^{\prime}$ has semiabelian reduction, and let $d$ be the ramification index of $K^{\prime}$ over $K$. Denote by $R^{\prime}$ the normalization of $R$ in $K^{\prime}$. Dualizing the morphism (2.3-1) and taking determinants, we find a morphism of free rank-one $R^{\prime}$-modules

$$
\operatorname{det}(\operatorname{Lie}(h))^{\vee}: \Omega_{G^{\prime}} \rightarrow \Omega_{G} \otimes_{R} R^{\prime}
$$

that induces an isomorphism

$$
\Omega_{G^{\prime}} \otimes_{R^{\prime}} K^{\prime} \cong \Omega_{G} \otimes_{R} K^{\prime}
$$


by tensoring with $K^{\prime}$. Thus, we can view $\Omega_{G}$ as a sub- $R$-module of $\Omega_{G^{\prime}} \otimes_{R^{\prime}} K^{\prime}$. This yields the following alternative description of the base change conductor:

Proposition 3.3.1. Let $\varpi^{\prime}$ be a uniformizer in $R^{\prime}$. The base change conductor $c(G)$ of $G$ is the unique element $r$ of $\mathbb{Z}[1 / d]$ such that

$$
\left(\varpi^{\prime}\right)^{r d} \omega_{G}
$$

generates the $R^{\prime}$-module $\Omega_{G^{\prime}}$.

Proof. Denote by ${ }^{\prime}{ }^{\prime}$ the Néron lft-model of $G^{\prime}$. By definition, the length of the cokernel of the natural morphism

$$
\operatorname{Lie}(h): \operatorname{Lie}\left(\mathscr{G} \times_{R} R^{\prime}\right) \rightarrow \operatorname{Lie}\left(\mathscr{G}^{\prime}\right)
$$

from (2.3-1) is equal to $c(G) d$. Writing $\operatorname{Lie}(h)$ in Smith normal form, it is easily seen that the cokernel of

$$
\operatorname{det}(\operatorname{Lie}(h))^{\vee}: \Omega_{G^{\prime}} \rightarrow \Omega_{G} \otimes_{R} R^{\prime}
$$

is isomorphic to $R^{\prime} /\left(\varpi^{\prime}\right)^{c(G) d}$.

Proposition 3.3.2. Let $R \rightarrow S$ be a flat local homomorphism of discrete valuation rings of ramification index one (in the sense of [Bosch et al. 1990, 3.6.1]), and denote by $L$ the quotient field of $S$. We denote by $g^{L}$ the Néron lft-model of $G \times{ }_{K} L$.

(1) The natural morphism

$$
\mathscr{G} \times_{R} S \rightarrow \mathscr{G}^{L}
$$

is an isomorphism. In particular, it induces an isomorphism of S-modules

$$
\Omega_{G} \otimes_{R} S \cong \Omega_{G \times{ }_{K} L} .
$$

(2) We have $c\left(G \times{ }_{K} L\right)=c(G)$.

This applies in particular to the case $S=\widehat{R^{s h}}$.

Proof. (1) The formation of Néron lft-models commutes with the base change $R \rightarrow S$ by [Bosch et al. 1990, 3.6.1].

(2) This follows easily from (1).

The semiabelian $K$-variety $G$ is bounded if and only if its Néron lft-model $\mathscr{G}$ is of finite type over $R$ [Bosch et al. 1990, 10.2.1]. In that case, $\mathscr{G}$ is called the Néron model of $G$. If $G$ is bounded, then for every gauge form $\omega$ on $G$, we can consider the motivic integral

$$
\int_{G}|\omega| \in M_{k}^{R}
$$

In particular, we can consider the motivic integral of the "motivic Haar measure" $\left|\omega_{G}\right|$ associated to $G$. It does not depend on the choice of $\omega_{G}$ since $\omega_{G}$ is unique up to multiplication with a unit in $R$. 
Proposition 3.3.3. Let $G$ be a bounded semiabelian $K$-variety of dimension $g$ with Néron model $G$. Let $\varpi$ be a uniformizer in $R$. Then for every integer $\gamma$, we have

$$
\int_{G}\left|\varpi^{\gamma} \omega_{G}\right|=\mathbb{L}^{-\gamma-g}\left[\mathscr{G}_{k}\right]
$$

in $M_{k}^{R}$. In particular, the virtual dimension of this motivic integral is equal to $-\gamma$. Proof. Since $\omega_{G}$ generates $\Omega_{G}$, we have

$$
\operatorname{ord}_{C}\left(\varpi^{\gamma} \omega_{G}\right)=\gamma
$$

for every connected component $C$ of $\varphi_{k}$. Thus, formula (3.2-1) becomes

$$
\int_{G}\left|\varpi^{\gamma} \omega_{G}\right|=\mathbb{L}^{-\gamma-g} \sum_{C \in \varpi_{0}\left(\mathscr{G}_{k}\right)}[C]=\mathbb{L}^{-\gamma-g}\left[\mathscr{G}_{k}\right]
$$

in $\mathcal{M}_{k}^{R}$, where the last equality follows from the scissor relations in the Grothendieck ring.

3.4. Split subtori and bounded varieties. We mentioned in Section 3.3 that a semiabelian $K$-variety $G$ is bounded if and only if the Néron lft-model $\varphi$ of $G$ is quasicompact. If $R$ is excellent (e.g., complete) and $k$ algebraically closed, then this is also equivalent to the property that $G$ does not contain a split torus [Bosch et al. 1990, 10.2.1]. Since the boundedness condition plays an important role in the definition of the motivic integral, we'll now take a closer look at split subtori of semiabelian varieties. The results in this section will allow us to establish an equivalence between Question 2.4.1 and a Fubini property of motivic integrals (Theorem 4.2.1).

Let $F$ be any field. We denote by $(\mathrm{SpT} / F)$ the category of split $F$-tori and $(\mathrm{SAb} / F)$ the category of semiabelian $F$-varieties (the morphisms in these categories are morphisms of algebraic $F$-groups).

For every semiabelian $F$-variety $G$, we denote by $G_{\text {sp }}$ the maximal split subtorus of $G$ [Halle and Nicaise 2010, 3.6]. If $T$ is a split $F$-torus, then every morphism of $F$-groups $T \rightarrow G$ factors through $G_{\text {sp }}$ by [Halle and Nicaise 2010, 3.5]. Thus, we can define a functor

$$
(\cdot)_{\mathrm{sp}}:(\mathrm{SAb} / F) \rightarrow(\mathrm{SpT} / F): G \mapsto G_{\mathrm{sp}} .
$$

For every semiabelian $F$-variety $G$, we put $G^{\mathrm{b}}=G / G_{\mathrm{sp}}$. Then $\left(G^{\mathrm{b}}\right)_{\mathrm{sp}}$ is trivial by the remark after [Halle and Nicaise 2010,3.6]. It follows that every morphism of semiabelian $F$-varieties $f: G \rightarrow H$ induces a morphism of semiabelian $F$-varieties

$$
f^{\mathrm{b}}: G^{\mathrm{b}} \rightarrow H^{\mathrm{b}}
$$

so that we obtain a functor

$$
(\cdot)^{\mathrm{b}}:(\mathrm{SAb} / F) \rightarrow(\mathrm{SAb} / F): G \mapsto G^{\mathrm{b}} .
$$


Lemma 3.4.1. Let $F$ be a field, and let $f: G \rightarrow H$ be a smooth morphism of semiabelian $K$-varieties. Then the morphism $f_{\mathrm{sp}}: G_{\mathrm{sp}} \rightarrow H_{\mathrm{sp}}$ is surjective.

Proof. The identity component of $G \times{ }_{H} H_{\mathrm{sp}}$ is a smooth and connected closed subgroup of $G$ and thus a semiabelian $F$-variety [Halle and Nicaise 2011, 5.2]. The morphism

$$
\left(G \times{ }_{H} H_{\mathrm{sp}}\right)^{o} \rightarrow H_{\mathrm{sp}}
$$

is still smooth. Therefore, we may assume that $H$ is a split torus. It follows from [Demazure and Grothendieck 1970a, $\mathrm{VI}_{\mathrm{B}} .1 .2$ ] that the image of $f$ is closed in $H$, and it is also open by flatness of $f$. Thus, $f$ is surjective.

We denote by $I$ the schematic image of the morphism $g: G_{\mathrm{sp}} \rightarrow H$. This is a closed subgroup of the split torus $H$. The quotient $H / I$ is again a split $F$ torus (it is geometrically connected because $G_{\mathrm{sp}}$ is geometrically connected, and it is smooth [Demazure and Grothendieck 1970a, $\mathrm{VI}_{\mathrm{B}} \cdot 9.2$ (xii)] and diagonalizable [Demazure and Grothendieck 1970b, IX.8.1] so that it is a split torus). The quotient $Q=G / G_{\text {sp }}$ is an extension of an abelian $F$-variety and an anisotropic $F$-torus so that the morphism of $F$-groups $Q \rightarrow H / I$ induced by $f$ is trivial. But this morphism is surjective by surjectivity of $f$ so that $H / I$ must be trivial and $H=I$. Since the image of $G_{\mathrm{sp}} \rightarrow H$ is closed [Demazure and Grothendieck 1970a, $\mathrm{VI}_{\mathrm{B}} .1 .2$ ], it follows that $G_{\mathrm{sp}} \rightarrow H$ is surjective.

Proposition 3.4.2. Let $F$ be a field, and let

$$
0 \rightarrow G_{1} \rightarrow G_{2} \rightarrow G_{3} \rightarrow 0
$$

be an exact sequence of semiabelian $F$-varieties.

(1) The schematic image of $\left(G_{1}\right)^{\mathrm{b}} \rightarrow\left(G_{2}\right)^{\mathrm{b}}$ is a semiabelian subvariety $H$ of $G_{2}$, and the morphism $\left(G_{1}\right)^{\mathrm{b}} \rightarrow H$ is an isogeny. Moreover, the sequence

$$
0 \rightarrow H \rightarrow\left(G_{2}\right)^{\mathrm{b}} \rightarrow\left(G_{3}\right)^{\mathrm{b}} \rightarrow 0
$$

is exact.

(2) If $\left(G_{3}\right)_{\mathrm{sp}}$ is trivial, then

$$
0 \rightarrow\left(G_{1}\right)^{\mathrm{b}} \rightarrow\left(G_{2}\right)^{\mathrm{b}} \rightarrow\left(G_{3}\right)^{\mathrm{b}} \rightarrow 0
$$

is exact.

Proof. Dividing $G_{1}$ and $G_{2}$ by $\left(G_{1}\right)_{\text {sp }}$, we may assume that $\left(G_{1}\right)_{\text {sp }}$ is trivial (here we use that $G^{\mathrm{b}}=(G / T)^{\mathrm{b}}$ for every semiabelian $F$-variety $G$ and every split subtorus $T$ of $G)$. Then $\left(G_{1}\right)^{\mathrm{b}}=G_{1}$.

First, we prove (1). The kernel of the morphism $G_{1} \rightarrow\left(G_{2}\right)^{\mathrm{b}}$ is the closed subgroup $\widetilde{G}_{1}=G_{1} \times G_{2}\left(G_{2}\right)_{\text {sp }}$ of $G_{1}$. It is also a closed subgroup of $\left(G_{2}\right)_{\text {sp. }}$. By [Demazure and Grothendieck $1970 \mathrm{a}, \mathrm{VI}_{\mathrm{B}} \cdot 9.2$ (xii)], the quotient $H=G_{1} / \widetilde{G}_{1}$ is 
smooth over $F$. Since $\left(G_{2}\right)_{\text {sp }}$ is a split $F$-torus, we know that $\widetilde{G}_{1}$ is a diagonalizable $F$-group [Demazure and Grothendieck 1970b, IX.8.1]. Since $\left(G_{1}\right)_{\mathrm{sp}}$ is trivial, the $F$-group $\widetilde{G}_{1}$ must be finite so that the projection $G_{1} \rightarrow H$ is an isogeny. The morphism $G_{1} \rightarrow G_{2}$ induces a morphism of $F$-groups $H \rightarrow\left(G_{2}\right)^{\mathrm{b}}$ that is a closed immersion [Demazure and Grothendieck $1970 \mathrm{a}, \mathrm{VI}_{\mathrm{B}}$.1.4.2]. It identifies $H$ with the schematic image of $G_{1} \rightarrow\left(G_{2}\right)^{\mathrm{b}}$. It follows from [Halle and Nicaise 2011, 5.2] that $H$ is a semiabelian $F$-variety because it is a connected smooth closed subgroup of the semiabelian $F$-variety $\left(G_{2}\right)^{\mathrm{b}}$.

It is clear that (3.4-1) is exact at the right so that it remains to prove that this sequence is also exact in the middle. By the natural isomorphism

$$
\left(G_{2} / G_{1}\right) /\left(\left(G_{2}\right)_{\mathrm{sp}} / \widetilde{G}_{1}\right) \cong\left(G_{2} /\left(G_{2}\right)_{\mathrm{sp}}\right) /\left(G_{1} / \widetilde{G}_{1}\right),
$$

it is enough to show $\widetilde{G}_{2}=\left(G_{2}\right)_{\mathrm{sp}} / \widetilde{G}_{1}$ is the maximal split subtorus of $G_{3}=G_{2} / G_{1}$. But $\left(G_{2}\right)_{\mathrm{sp}} \rightarrow\left(G_{3}\right)_{\mathrm{sp}}$ is surjective by Lemma 3.4.1, and its kernel is precisely $\widetilde{G}_{1}$, so we see that $\widetilde{G}_{2}=\left(G_{3}\right)_{\mathrm{sp}}$.

Now we prove (2). Assume that $\left(G_{3}\right)_{\mathrm{sp}}$ is trivial. Then the closed immersion $\left(G_{2}\right)_{\mathrm{sp}} \rightarrow G_{2}$ factors through $G_{1}$, and since $\left(G_{1}\right)_{\mathrm{sp}}$ is trivial, we find that $\left(G_{2}\right)_{\mathrm{sp}}$ must be trivial. Thus, $G_{i}=\left(G_{i}\right)^{\mathrm{b}}$ for $i=1,2,3$, and the result is obvious.

If $\left(G_{3}\right)_{\mathrm{sp}}$ is not trivial, it can happen that the sequence

$$
0 \rightarrow\left(G_{1}\right)^{\mathrm{b}} \rightarrow\left(G_{2}\right)^{\mathrm{b}} \rightarrow\left(G_{3}\right)^{\mathrm{b}} \rightarrow 0
$$

in Proposition 3.4.2 is not left exact as is shown by the following:

Example 3.4.3. Let $K$ be the field $\mathbb{C}((t))$ of complex Laurent series, and put $K^{\prime}=K((\sqrt{t}))$. The Galois group $\Gamma=\operatorname{Gal}\left(K^{\prime} / K\right)$ is isomorphic to $\mathbb{Z} / 2 \mathbb{Z}$, and it is generated by the automorphism $\sigma$ that maps $\sqrt{t}$ to $-\sqrt{t}$. Let $G_{2}$ be the $K$-torus with splitting field $K^{\prime}$ and character module

$$
X\left(G_{2}\right)=\mathbb{Z} \cdot e_{1} \oplus \mathbb{Z} \cdot e_{2}
$$

where $\sigma$ permutes $e_{1}$ and $e_{2}$.

Let $G_{1}$ be the maximal anisotropic subtorus of $G_{2}$. Its character module is $X\left(G_{1}\right)=X\left(G_{2}\right) / X\left(G_{2}\right)^{\Gamma}$. We put $G_{3}=G_{2} / G_{1}$. This is a split $K$-torus with character module $X\left(G_{3}\right)=X\left(G_{2}\right)^{\Gamma}=\mathbb{Z} \cdot\left(e_{1}+e_{2}\right)$.

For every $K$-torus $T$ that splits over $K^{\prime}$, we can consider the trace map

$$
\operatorname{tr}_{T}: X(T) \rightarrow X(T)^{\Gamma}: x \mapsto x+\sigma \cdot x .
$$

It follows from the duality between tori and their character modules that the maximal split subtorus of $T$ has character module $X(T) / \operatorname{ker}\left(\operatorname{tr}_{T}\right)$ and that $T^{\mathrm{b}}$ is the $K$-torus with character module $\operatorname{ker}\left(\operatorname{tr}_{T}\right)$. In this way, we see that $\left(G_{1}\right)_{\mathrm{sp}}$ is trivial and that 
$\left(G_{2}\right)^{\mathrm{b}}$ is the $K$-torus with character module

$$
\operatorname{ker}\left(\operatorname{tr}_{G_{2}}\right)=\mathbb{Z} \cdot\left(e_{1}-e_{2}\right) .
$$

Thus, applying the functor $(\cdot)^{\mathrm{b}}$ to the exact sequence of $K$-tori

$$
0 \rightarrow G_{1} \rightarrow G_{2} \rightarrow G_{3} \rightarrow 0
$$

we obtain the sequence

$$
0 \rightarrow G_{1} \rightarrow\left(G_{2}\right)^{\mathrm{b}} \rightarrow 0 \rightarrow 0
$$

and the morphism of $K$-tori $G_{1} \rightarrow\left(G_{2}\right)^{\mathrm{b}}$ corresponds to the morphism of character modules

$$
\alpha: \mathbb{Z} \cdot\left(e_{1}-e_{2}\right) \rightarrow X\left(G_{2}\right) / X\left(G_{2}\right)^{\Gamma} .
$$

The morphism $\alpha$ is injective but not surjective; its cokernel is

$$
X\left(G_{2}\right) /\left(\mathbb{Z} \cdot\left(e_{1}-e_{2}\right)+\mathbb{Z} \cdot\left(e_{1}+e_{2}\right)\right) \cong \mathbb{Z} / 2 \mathbb{Z}
$$

with trivial $\Gamma$-action. Therefore, (3.4-3) is not exact. More precisely, the morphism

$$
G_{1} \rightarrow\left(G_{2}\right)^{\mathrm{b}}
$$

is an isogeny with kernel $\mu_{2, K}$.

Proposition 3.4.4. Assume that $R$ is excellent and that $k$ is algebraically closed. For every semiabelian $K$-variety $G$, the quotient $G^{\mathrm{b}}$ is a bounded semiabelian $K$-variety.

Proof. Since $\left(G^{\mathrm{b}}\right)_{\mathrm{sp}}$ is trivial, this follows immediately from [Bosch et al. 1990, 10.2.1].

\section{Chai's conjecture and Fubini properties of motivic integrals}

\subsection{Chai's conjecture and Haar measures. Let}

$$
0 \rightarrow T \rightarrow G \rightarrow A \rightarrow 0
$$

be a short exact sequence of semiabelian $K$-varieties (as the notation suggests, the main example we have in mind is the Chevalley decomposition of a semiabelian $K$-variety $G$ as in Conjecture 1.1, but we will work in greater generality). The sequence of $K$-vector spaces

$$
0 \rightarrow \operatorname{Lie}(T) \rightarrow \operatorname{Lie}(G) \rightarrow \operatorname{Lie}(A) \rightarrow 0
$$

is exact, and by dualizing and taking determinants, we find a canonical isomorphism of $K$-vector spaces

$$
\Omega_{G} \otimes_{R} K \cong\left(\Omega_{T} \otimes_{R} \Omega_{A}\right) \otimes_{R} K
$$


In this way, we can view $\Omega_{T} \otimes_{R} \Omega_{A}$ as an $R$-lattice in $\Omega_{G} \otimes_{R} K$.

The following proposition is implicit in the proof on pages 724-725 of [Chai 2000] (proof of Proposition 4.1 in [loc. cit.] when the residue field is finite):

Proposition 4.1.1. Assume that $T$ is a torus. Let $\omega_{T}$ and $\omega_{A}$ be generators of $\Omega_{T}$ and $\Omega_{A}$, respectively. Let $\varpi$ be a uniformizer of $R$, and denote by $\gamma$ the unique integer such that $\varpi^{-\gamma}\left(\omega_{T} \otimes \omega_{A}\right)$ generates the $R$-module $\Omega_{G}$. Then

$$
c(G)=c(T)+c(A)+\gamma .
$$

In particular, $c(G)-c(T)-c(A)$ belongs to $\mathbb{Z}$.

Proof. By Proposition 3.3.2, we may assume that $R$ is complete and that $k$ is algebraically closed. Suppose that

$$
\omega_{G}:=\varpi^{-\gamma}\left(\omega_{T} \otimes \omega_{A}\right)
$$

generates $\Omega_{G}$. Let $K^{\prime}$ be a finite separable extension of $K$ such that $G^{\prime}=G \times{ }_{K} K^{\prime}$ has semiabelian reduction, and denote by $R^{\prime}$ the normalization of $R$ in $K^{\prime}$. Then $A^{\prime}=A \times_{K} K^{\prime}$ has semiabelian reduction and $T^{\prime}=T \times_{K} K^{\prime}$ is split [Halle and Nicaise 2010, 4.1].

We denote by $\varpi^{\prime}$ a uniformizer of $R^{\prime}$ and $d$ the ramification degree of the extension $K^{\prime} / K$. By Proposition 3.3.1, the $R^{\prime}$-module $\Omega_{G^{\prime}}$ is generated by

$$
\left(\varpi^{\prime}\right)^{c(G) d} \omega_{G},
$$

and the analogous property holds for $A$ and $T$. We denote by $\mathscr{G}^{\prime}, \mathscr{T}^{\prime}$ and $\mathscr{A}^{\prime}$ the Néron lft-models of $G^{\prime}, T^{\prime}$ and $A^{\prime}$, respectively. By the universal property of the Néron lft-model, the exact sequence

$$
0 \rightarrow T^{\prime} \rightarrow G^{\prime} \rightarrow A^{\prime} \rightarrow 0
$$

extends uniquely to a sequence of $R^{\prime}$-group schemes

$$
0 \rightarrow \mathscr{T}^{\prime} \rightarrow \mathscr{G}^{\prime} \rightarrow \mathscr{A}^{\prime} \rightarrow 0
$$

and this sequence is exact by [Chai 2000, 4.8(a)]. It follows that

$$
\Omega_{G^{\prime}}=\Omega_{T^{\prime}} \otimes_{R^{\prime}} \Omega_{A^{\prime}} \subset \Omega_{G^{\prime}} \otimes_{R^{\prime}} K^{\prime}
$$

so that both $\left(\varpi^{\prime}\right)^{c(G) d} \omega_{G}$ and

$$
\left(\varpi^{\prime}\right)^{(c(T)+c(A)) d}\left(\omega_{T} \otimes \omega_{A}\right)=\left(\varpi^{\prime}\right)^{(c(T)+c(A)) d} \varpi^{\gamma} \omega_{G}
$$

are generators of the free $R^{\prime}$-module $\Omega_{G^{\prime}}$. Thus, we find that

$$
\left(\varpi^{\prime}\right)^{(c(G)-c(T)-c(A)) d} \varpi^{-\gamma}
$$


is a unit in $R^{\prime}$. This means that its $\varpi^{\prime}$-adic valuation is zero so that

$$
c(G)=c(T)+c(A)+\gamma .
$$

Remark 4.1.2. Let

$$
0 \rightarrow T \rightarrow G \rightarrow A \rightarrow 0
$$

be an exact sequence of semiabelian $K$-varieties, and let $\omega_{T}$ and $\omega_{A}$ be generators of $\Omega_{T}$ and $\Omega_{A}$, respectively. Chai [2000, §8.1] considers the following statement:

$$
\text { One has } c(G)=c(T)+c(A) \text { if and only if } \omega_{T} \otimes \omega_{A} \text { generates } \Omega_{G} \text {. }
$$

If $T$ is a torus, then this is a corollary of Proposition 4.1.1. However, if $T$ is not a torus, it is not clear to us how statement $(*)$ can be proven although Chai hints that it may be implicit in the proof on pages 724-725 of [Chai 2000]. If $G, T$ and $A$ have semiabelian reduction, then $c(G)=c(T)=c(A)=0$ so that statement $(*)$ contains the following special case:

If $G, T$ and $A$ have semiabelian reduction, then $\omega_{T} \otimes \omega_{A}$ generates $\Omega_{G} . \quad(* *)$

Even this property does not seem obvious because the sequence of identity components of Néron lft-models

$$
0 \rightarrow \mathscr{T}^{o} \rightarrow \mathscr{G}^{o} \rightarrow \mathscr{A}^{o} \rightarrow 0
$$

might not be exact; see [Bosch et al. 1990, 7.5.8] for an example where $T, G$ and $A$ are abelian varieties with good reduction and $\mathscr{T}^{\circ} \rightarrow \mathscr{G}^{\circ}$ is not a monomorphism. If statement $(* *)$ is true, then the proof of Proposition 4.1.1 shows that Proposition 4.1.1, and thus statement $(*)$, are valid without the assumption that $T$ is a torus.

Lemma 4.1.3. Let $G$ be a semiabelian $K$-variety, and let $T$ be a split subtorus of $G$. Then $c(G)=c(G / T)$. In particular, $c(G)=c\left(G^{\mathrm{b}}\right)$.

Proof. We set $H=G / T$. By [Chai 2000, 4.8(a)], the canonical sequence of group schemes

$$
0 \rightarrow \mathscr{T} \rightarrow \mathscr{G} \rightarrow \mathscr{H} \rightarrow 0
$$

is exact so that $\Omega_{T} \otimes \Omega_{H}=\Omega_{G}$. Now the result follows from Proposition 4.1.1 and the fact that $c(T)=0$ because $T$ has semiabelian reduction.

4.2. Main results. We can now state our main results.

Theorem 4.2.1. Let

$$
0 \rightarrow T \rightarrow G \rightarrow A \rightarrow 0
$$


be an exact sequence of semiabelian $K$-varieties with $T$ a torus. We put

$$
\widetilde{G}=\left(G \times_{K} \widehat{K^{s h}}\right)^{\mathrm{b}} \text { and } \tilde{A}=\left(A \times_{K} \widehat{K^{s h}}\right)^{\mathrm{b}},
$$

and we denote by $\widetilde{T}$ the schematic image of the morphism

$$
\left(T \times_{K} \widehat{K^{s h}}\right)^{\mathrm{b}} \rightarrow \widetilde{G} .
$$

Then $\widetilde{T}$ is a $\widehat{K^{s h}}$-subtorus of $\widetilde{G}$,

$$
0 \rightarrow \widetilde{T} \rightarrow \widetilde{G} \rightarrow \widetilde{A} \rightarrow 0
$$

is an exact sequence of bounded semiabelian $\widehat{K^{s h}}$-varieties and

$$
c(G)=c(T)+c(A)
$$

if and only if

$$
\operatorname{dim} \int_{\widetilde{G}}\left|\omega_{\widetilde{T}} \otimes \omega_{\widetilde{A}}\right|=\operatorname{dim} \int_{\widetilde{T}}\left|\omega_{\widetilde{T}}\right|+\operatorname{dim} \int_{\widetilde{A}}\left|\omega_{\widetilde{A}}\right|=0 .
$$

Proof. By Proposition 3.3.2, we may assume that $K$ is complete and $k$ algebraically closed so that $\widehat{K^{s h}}=K$. By Proposition 3.4.2, we know that $T^{\mathrm{b}}$ and $\widetilde{T}$ are isogenous $K$-tori so that $c\left(T^{\mathrm{b}}\right)=c(\widetilde{T})$ by [Chai and Yu 2001, 11.3 and 12.1]. Thus, by Proposition 3.4.2 and Lemma 4.1.3, we may assume that $\widetilde{T}=T, \widetilde{G}=G$ and $\widetilde{A}=A$. Then $T, G$ and $A$ are bounded, by Proposition 3.4.4, so that we can take motivic integrals of gauge forms on $T, G$ and $A$.

Let $\varpi$ be a uniformizer in $R$. It follows from Proposition 3.3.3 that

$$
\operatorname{dim} \int_{T}\left|\omega_{T}\right|+\operatorname{dim} \int_{A}\left|\omega_{A}\right|=0
$$

and that

$$
\operatorname{dim} \int_{G}\left|\omega_{T} \otimes \omega_{A}\right|
$$

is equal to the unique integer $\gamma$ such that

$$
\varpi^{\gamma}\left(\omega_{T} \otimes \omega_{A}\right)
$$

generates the $R$-module $\Omega_{G}$. By Proposition 4.1.1, we know that $\gamma=0$ if and only if $c(G)=c(T)+c(A)$.

Remark 4.2.2. In Conjecture $1.1, T$ is a torus and $A$ is an abelian variety. This implies that $A_{\text {sp }}$ is trivial so that $A=A^{\mathrm{b}}$ and

$$
0 \rightarrow T^{\mathrm{b}} \rightarrow G^{\mathrm{b}} \rightarrow A \rightarrow 0
$$

is exact by Proposition 3.4.2. In this case, in the proof of Theorem 4.2.1, we do not need the fact that the base change conductor of a torus is invariant under isogeny. 
Theorem 4.2.3. Assume that $K$ is complete and of characteristic zero and that $k$ is algebraically closed. Let

$$
0 \rightarrow T \rightarrow G \rightarrow A \rightarrow 0
$$

be an exact sequence of bounded semiabelian $K$-varieties with $T$ a torus. Then

$$
\operatorname{dim} \int_{G}\left|\omega_{T} \otimes \omega_{A}\right|=\operatorname{dim} \int_{T}\left|\omega_{T}\right|+\operatorname{dim} \int_{A}\left|\omega_{A}\right|=0 .
$$

We will prove Theorem 4.2.3 in Section 5.4, using the model-theoretic approach to motivic integration in [Cluckers and Loeser 2012] and a new result on dimensions of motivic integrals (Theorem 5.2.1). As a corollary, we obtain a new proof of the following theorem:

Theorem 4.2.4 (Chai). Let

$$
0 \rightarrow T \rightarrow G \rightarrow A \rightarrow 0
$$

be an exact sequence of semiabelian $K$-varieties with $T$ a torus. Assume that $K$ is of characteristic zero. Then

$$
c(G)=c(T)+c(A) .
$$

Proof. This follows at once from Theorem 4.2.1 and Theorem 4.2.3.

Remark 4.2.5. Theorem 4.2.3 is not a direct corollary of the Fubini theorem in [Cluckers and Loeser 2012] and the above results. We need to combine the Fubini result [Cluckers and Loeser 2012, 12.5] with the new result in Theorem 5.2.1 and its corollary below, which compares dimensions of motivic parameter integrals under rather general conditions. By the lack of a definable section for the morphism $G \rightarrow A$ as in Theorem 4.2.3, the motivic integral of $\left|\omega_{G}\right|$ over $G$ may not be equal to the product of the integrals of $\left|\omega_{T}\right|$ over $T$ and of $\left|\omega_{A}\right|$ over $A$. By the corollary to Theorem 5.2.1 and by the change of variables in [Cluckers and Loeser 2012, 12.4], this product survives at the rough level of virtual dimensions, which is sufficient to prove Theorem 4.2.3.

\section{A comparison result for the dimensions of motivic integrals}

In this section, we will work with a specific context falling under [Cluckers and Loeser 2012] and define the dimension of motivic constructible functions at each point. These functions play an important role in motivic integration and in general Fubini results of [Cluckers and Loeser 2012; 2008]. In order to control dimensions as desired for Equation (4.2-1) in Theorem 4.2.1, we will compare the dimensions of the integrals of possibly different functions $F$ and $G$ when we are given that $F$ and $G$ have the same dimension in every point. Theorem 5.2.1 provides such 
a comparison result with parameters, and its corollary gives a similar comparison result for integrals on an algebraic variety with a volume form.

5.1. Dimensions of motivic constructible functions. In this section, we suppose $R$ is a complete discrete valuation ring of characteristic zero with quotient field $K$ and algebraically closed residue field $k$ of characteristic $p \geq 0$. We fix a uniformizer $\varpi$ in $K$. We will use some terminology and results from [Cluckers and Loeser 2012] with precise references. Let $\mathscr{L}_{\text {high }}(K)$ be the Denef-Pas language $\mathscr{L}_{\text {high }}$ as in [Cluckers and Loeser 2012, §2.3] enriched with coefficients from $K$ and where the angular component maps $\overline{\mathrm{ac}}_{n}$ for $n>0$ are given by $\overline{\mathrm{ac}}_{n}(x)=x \varpi^{-\operatorname{ord} x} \bmod (\varpi)^{n}$ for nonzero $x \in K$. Let $\mathscr{T}$ be the $\mathscr{L}_{\text {high }}(K)$-theory of $K$.

Since $\mathcal{T}$ falls under the combined Examples 1 and 4 of [Cluckers and Loeser 2012, §3.1], we can use the theory of motivic integration of [Cluckers and Loeser 2012]. Also, since $\mathcal{T}$ is a complete theory, any definable subassignment $X$ is uniquely determined by the definable set $X(K)$ with notation from [Cluckers and Loeser 2012, §4.1]. We will sometimes say "definable set" instead of "definable subassignment".

We first define how to take (virtual) dimensions of several objects appearing in [Cluckers and Loeser 2012]. Write $R_{n}$ for the ring $R /\left(\varpi^{n}\right)$ and $\varpi_{n}$ for the image of $\varpi$ in $R_{n}$. Let $\mathscr{L}_{r}$ be the multisorted language with sorts $R_{n}$ for integers $n>0$, on each $R_{n}$ the ring language with coefficients from $R_{n}$, and with the natural projection maps $p_{n, m}: R_{n} \rightarrow R_{m}$ for $n \geq m$. It follows from the quantifier elimination results of $[$ Pas $1989 ; 1990]$ that any $\mathscr{L}_{\text {high }}(K)$-definable set $X \subset \prod_{i=1}^{s} R_{n_{i}}$ is already $\mathscr{L}_{r}$ definable with parameters; see also [Cluckers and Loeser 2012, Theorem 3.10]. To each $\mathscr{L}_{r}$-definable set $X \subset \prod_{i=1}^{s} R_{n_{i}}$, we associate an $\mathscr{L}_{\text {ring }}(k)$-definable set $\delta(X)$ as follows. If $R$ has mixed characteristic, the projection $p_{n, 1}$ induces a bijection from the set of $p^{n}$-th powers in $R_{n}$ to $k$ by Hensel's lemma, Newton's binomial theorem and the hypotheses on $K$. Let us write $P_{p^{n}}$ for the set of $p^{n}$-th powers in $R_{n}$. Then any $x$ in $R_{n}$ can be written uniquely as

$$
\sum_{i=0}^{n-1} x_{i} \varpi_{n}^{i},
$$

with $x_{i} \in P_{p^{n}}$, yielding a bijection $R_{n} \rightarrow k^{n}: x \mapsto\left(p_{n, 1}\left(x_{i}\right)\right)_{i}$ that is, in fact, $\mathscr{L}_{r}$-definable. If $R$ has equal characteristic zero, we choose a retraction $k \rightarrow R$ of the ring morphism $R \rightarrow k$. This choice determines an isomorphism $R \rightarrow k \llbracket \varpi \rrbracket$, and we identify $R_{n} \cong k[\varpi] /\left(\varpi^{n}\right)$ with the $k$-vector space $k^{n}$ by means of the basis $1, \varpi, \ldots, \varpi^{n-1}$ of $R_{n}$. In both cases, we obtain a bijection $\prod_{i=1}^{s} R_{n_{i}} \rightarrow k^{N}$ with $N=\sum_{i=1}^{s} n_{i}$. This identification maps the $\mathscr{L}_{r}$-definable subset $X$ of $\prod_{i=1}^{s} R_{n_{i}}$ onto an $\mathscr{L}_{\text {ring }}(k)$-definable subset of $k^{N}$ that we denote by $\delta(X)$. 
Recall from [Cluckers and Loeser 2012, §7.1] that $\mathscr{C}_{+}$(Point) is the Grothendieck semiring of $\mathscr{L}_{\text {high }}(K)$-definable subsets of Cartesian products of the form $\prod_{i=1}^{s} R_{n_{i}}$ up to definable isomorphisms with scissor relations, with zero-element $[\varnothing]$ and localized with respect to $\mathbb{L}$ and the elements $\mathbb{L}^{i}-1$ for all $i>0$, where $\mathbb{L}$ stands for the class of the affine line over $k$. Clearly, $\delta$ induces a semiring morphism

$$
\mathscr{C}_{+}(\text {Point }) \rightarrow \mathcal{M}_{k}^{+},
$$

which we also denote by $\delta$. Recall that objects in $\mu_{k}^{+}$have a dimension by Definition 3.1.1.

For an $\mathscr{L}_{\text {high }}(K)$-definable set $Z, \mathscr{C}_{+}(Z)$ is a relative variant of $\mathscr{C}_{+}$(Point) over $Z$; see [Cluckers and Loeser 2012, §7.1]. An object $\varphi \in \mathscr{C}_{+}(Z)$ is called a motivic constructible function on $Z$. Moreover, for every $z \in Z(K)$, there is the evaluation map $i_{z}^{*}: \mathscr{C}_{+}(Z) \rightarrow \mathscr{C}_{+}$(Point) at $z$, and $i_{z}^{*}(\varphi)$ is called the evaluation of $\varphi$ at $z$. For an $\mathscr{L}_{\text {high }}(K)$-definable set $Z$, a point $z \in Z(K)$ and a function $\varphi$ in $\mathscr{C}_{+}(Z)$, the dimension of $\varphi$ at $z$ is defined as $\operatorname{dim}\left(\delta\left(i_{z}^{*}(\varphi)\right)\right)$ and is denoted by $\operatorname{dim}_{z}(\varphi)$. If $Z$ is the point and $\varphi \in \mathscr{C}_{+}$(Point), we write $\operatorname{dim}(\varphi)$ instead of $\operatorname{dim}_{\text {Point }}(\varphi)$.

5.2. A comparison result. In this section, definable will mean for the language $\mathscr{L}_{\text {high }}(K)$. Recall that, for definable sets $X, Y$ and $Z \subset X \times Y$, under integrability conditions in the fibers of the projection $Z \rightarrow X$, called $X$-integrability, one can integrate $\varphi \in \mathscr{C}_{+}(Z)$ in the fibers of the projection $Z \rightarrow X$ to obtain a function $\mu_{/ X}(\varphi)$ in $\mathscr{C}_{+}(X)$; see [Cluckers and Loeser 2012, 9.1]. The method of [Cluckers and Loeser 2008; 2012] for calculating integrals goes back to ideas by Denef [1984] in the $p$-adic case and to Pas $[1989 ; 1990]$ in a premotivic setting.

Now we can state and prove our comparison result, stating that the dimension of a motivic integral only depends on the dimensions of the values of the integrand at each point.

Theorem 5.2.1. Let $F$ and $G$ be in $\mathscr{C}_{+}(Z)$, and suppose that $Z \subset X \times Y$ for some definable sets $X, Y$ and $Z$. Suppose that $F$ and $G$ are $X$-integrable and that

$$
\operatorname{dim}_{z}(F)=\operatorname{dim}_{z}(G) \text { for each point } z \text { on } Z(K) .
$$

Then one has

$$
\operatorname{dim}_{x}\left(\mu_{/ X}(F)\right)=\operatorname{dim}_{x}\left(\mu_{/ X}(G)\right) \text { for each point } x \text { on } X(K) .
$$

Proof. For some integers $n, r, s \geq 0$ and for some tuple $m=\left(m_{1}, \ldots, m_{s}\right)$ of nonnegative integers, $Y$ is contained in $K^{n} \times \prod_{1 \leq i \leq s} R_{m_{i}} \times \mathbb{Z}^{r}$. By projecting one variable at a time and by iterating the one variable result, it suffices to consider the case where two of the three values $n, m$ and $r$ are zero and either $n=1, r=1$ or $s=1$ and $m_{1}=1$. By the cell decomposition theorem of [Pas 1989; 1990], we may suppose that $n=0$. Indeed, via cell decomposition, each integral over a valued 
field variable is precisely calculated as a sum over $\mathbb{Z}$-variables and a subsequent integral over residue ring variables; see [Cluckers and Loeser 2012, §8].

Recall that $F$ is a finite sum of terms of the form $a_{i} \otimes b_{i}$, with $a_{i} \in \mathscr{P}_{+}(Z)$ and $b_{i} \in 2_{+}(Z)$, and similarly for $G$ with notation from [Cluckers and Loeser 2012, §7.1]. (The semiring $\mathscr{P}_{+}(Z)$ is related to the value group and $\mathscr{2}_{+}(Z)$ to the residue field, and $\mathscr{C}_{+}(Z)$ is a tensor product of both.)

If $n=r=0$, then we may suppose that the $a_{i}$ lie in $\mathscr{P}_{+}(X)$, and similarly for $G$, by Proposition 7.5 of [Cluckers and Loeser 2012]. The result of the theorem now follows from the definition in [Cluckers and Loeser 2012, §6] of $\mu / X$ in this case and the following simple comparison property for dimensions of constructible sets $A_{i} \subset \mathbb{A}_{k}^{n_{i}}$. If, for certain morphisms $f_{i}: \mathbb{A}_{k}^{n_{i}} \rightarrow \mathbb{A}_{k}^{n_{3}}$ for $i=1,2$, one has that $f_{1}\left(A_{1}\right)=f_{2}\left(A_{2}\right)$ and, for each $x \in \mathbb{A}_{k}^{n_{3}}(k)$, the dimension of $f_{1}^{-1}(x) \cap A_{1}$ equals the dimension of $f_{2}^{-1}(x) \cap A_{2}$, then one has $\operatorname{dim}\left(A_{1}\right)=\operatorname{dim}\left(A_{2}\right)$.

Let us finally consider the case that $n=m=0$ and $r=1$. In this case, we may suppose that the $b_{i}$ lie in $2_{+}(X)$, and similarly for $G$, again by Proposition 7.5 of [Cluckers and Loeser 2012]. In the considered case, the theorem follows from the definition of $\mu_{/ X}$ of [Cluckers and Loeser 2012] and the following two observations. For any $a \in \mathbb{A}$, where $a=a(\mathbb{L})$ is thus a rational function in $\mathbb{L}$ of a specific kind, one has that $\operatorname{dim}(a)$ equals the degree of the rational function $a(\mathbb{L})$, where the degree of a rational function is the degree of its numerator minus the degree of its denominator and where the degree of 0 is defined as $-\infty$. Secondly, there is the following elementary comparison property for the degrees of rational functions. Consider, for each $i \in \mathbb{Z}$, an integer $n_{i} \geq 0$ and a polynomial $a_{i}(x)$ over $\mathbb{Z}$ in one variable $x$ such that $a_{i}(q) \geq 0$ for each real $q>1$. If there is a rational function $r(x)$ such that $\sum_{i \in \mathbb{Z}} a_{i}(q) / q^{n_{i}}$ converges and equals $r(q)$ for each real $q>1$, then

$$
\operatorname{deg}(r(x))=\max _{i} \operatorname{deg}\left(\frac{a_{i}(x)}{x^{n_{i}}}\right),
$$

where deg stands for the degree. Since summation of nonnegative functions over $\mathbb{Z}$ in [Cluckers and Loeser 2012, §5] is calculated and defined by considering specific sums of rational functions in $\mathbb{L}$ and by evaluating in real numbers $q>1$, the result follows.

By working with affine charts over $K$, one may consider a variety $V$ over $K$ as a definable subassignment, and one defines $\mathscr{C}_{+}(V)$ correspondingly; see [Cluckers and Loeser 2012, §12.3]. Let us write $\int^{\mathrm{CL}}$ for the integral as defined there, to distinguish them from the integrals from Section 3.2 of this paper. The definition of these integrals is based on finite affine covers of $V$ over $K$, on finite additivity for motivic integrals and on the change of variables formula.

Corollary 5.2.2. Let $V$ be an algebraic variety over $K$ with a volume form $\omega_{V}$. Let $F$ and $G$ be integrable functions in $\mathscr{C}_{+}(V)$ such that, for each $x \in V(K)$, one has 
$\operatorname{dim}_{x}(F)=\operatorname{dim}_{x}(G)$. Then their integrals have the same dimension:

$$
\operatorname{dim} \int_{V}^{\mathrm{CL}} F\left|\omega_{V}\right|=\operatorname{dim} \int_{V}^{\mathrm{CL}} G\left|\omega_{V}\right| .
$$

Proof. This follows immediately from Theorem 5.2.1 and the definition of the integrals $\int^{\mathrm{CL}}$ in [Cluckers and Loeser 2012, §12.3].

5.3. Gel'fand-Leray residues. Let $f: X \rightarrow Y$ be a smooth morphism between smooth equidimensional varieties over $K$. Let $m$ be the dimension of $Y$, and let $m+n$ be the dimension of $X$. Let $\omega_{X}$ and $\omega_{Y}$ be differential forms of maximal degree on $X$ and $Y$, respectively. Assume that $\omega_{Y}$ is a gauge form, that is, a generator of the line bundle $\Omega_{Y / K}^{m}$ at each point of $Y$.

Since $f$ is smooth, the fundamental sequence of locally free coherent $\mathrm{O}_{X}$-modules

$$
0 \rightarrow f^{*} \Omega_{Y / K}^{1} \rightarrow \Omega_{X / K}^{1} \rightarrow \Omega_{X / Y}^{1} \rightarrow 0
$$

is exact [Grothendieck and Dieudonné 1967, 17.2.3]. Taking maximal exterior powers, we obtain an isomorphism

$$
f^{*} \Omega_{Y / K}^{m} \otimes \Omega_{X / Y}^{n} \rightarrow \Omega_{X / K}^{m+n} .
$$

Locally, this isomorphism is defined by

$$
\varphi \otimes \eta \mapsto \varphi \wedge \tilde{\eta}
$$

where $\tilde{\eta}$ is any lift of $\eta$ to $\Omega_{X / K}^{n}$. Since $f^{*} \omega_{Y}$ generates the line bundle $f^{*} \Omega_{Y / K}^{m}$, we obtain an isomorphism $\Omega_{X / Y}^{n} \rightarrow \Omega_{X / K}^{m+n}$ that is locally defined by

$$
\eta \mapsto f^{*} \omega_{Y} \wedge \tilde{\eta}
$$

The inverse image of $\omega_{X}$ under this isomorphism is called the Gel'fand-Leray form associated to $\omega_{X}$ and $\omega_{Y}$ and denoted by $\omega_{X} / \omega_{Y}$. It induces a differential form of maximal degree on each of the fibers of $f$.

5.4. Proof of Theorem 4.2.3. It follows from Proposition 3.3 .3 that

$$
\operatorname{dim} \int_{T}\left|\omega_{T}\right|=\operatorname{dim} \int_{A}\left|\omega_{A}\right|=0 .
$$

It is proven in Proposition 12.6 of [Cluckers and Loeser 2012] that the theory of motivic integration developed there can be used to compute the motivic integrals defined by the formula (3.2-1). In particular, the dimensions of the respective motivic integrals are the same so that we can use the corollary of Theorem 5.2.1 and the results in [Cluckers and Loeser 2012] to prove Theorem 4.2.3.

We denote the projection morphism $G \rightarrow A$ by $f$. Since $H^{1}(K, T)=0$ by [Chai 2000, 4.3], the map $f(K): G(K) \rightarrow A(K)$ is surjective. For every $a \in A(K)$, 
we set $G_{a}=f^{-1}(a)$. If we choose a point $x$ in $G_{a}(K)$, then the multiplication by $x$ defines an isomorphism $\tau_{x}: T \rightarrow G_{a}$. Since the relative differential form $\left(\omega_{T} \otimes \omega_{A}\right) / \omega_{A}$ on $G$ is invariant under translation, the pullback through $\tau_{x}$ of its restriction to $G_{a}$ equals $\omega_{T}$. Thus, by the change of variables formula in [Cluckers and Loeser 2012, 12.4], we have

$$
\int_{G_{a}}^{\mathrm{CL}}\left|\left(\omega_{T} \otimes \omega_{A}\right) / \omega_{A}\right|=\int_{T}^{\mathrm{CL}}\left|\omega_{T}\right|
$$

for each $a$ in $A(K)$. Hence, by the Fubini property in [Cluckers and Loeser 2012, 12.5], we find that

$$
\int_{G}^{\mathrm{CL}}\left|\omega_{T} \otimes \omega_{A}\right|=\int_{A}^{\mathrm{CL}} \psi\left|\omega_{A}\right|
$$

where $\psi$ is a motivic constructible function on $A$ such that $\operatorname{dim}_{a}(\psi)=0$ for each $a \in A(K)$. Now Corollary 5.2.2 with $V=A$ implies that

$$
\operatorname{dim} \int_{A}^{C L} \psi\left|\omega_{A}\right|=\operatorname{dim} \int_{A}^{C L}\left|\omega_{A}\right|=0 .
$$

Combining the above equations, we find

$$
\operatorname{dim} \int_{G}\left|\omega_{T} \otimes \omega_{A}\right|=\operatorname{dim} \int_{G}^{C L}\left|\omega_{T} \otimes \omega_{A}\right|=0,
$$

which concludes the proof.

\section{References}

[Bosch et al. 1990] S. Bosch, W. Lütkebohmert, and M. Raynaud, Néron models, Ergebnisse der Mathematik und ihrer Grenzgebiete (3) 21, Springer, Berlin, 1990. MR 91i:14034 Zbl 0705.14001

[Chai 2000] C.-L. Chai, "Néron models for semiabelian varieties: Congruence and change of base field", Asian J. Math. 4:4 (2000), 715-736. MR 2002i:14025 Zbl 1100.14511

[Chai and Yu 2001] C.-L. Chai and J.-K. Yu, "Congruences of Néron models for tori and the Artin conductor", Ann. of Math. (2) 154:2 (2001), 347-382. MR 2003e:11126 Zbl 1098.14014

[Cluckers and Loeser 2008] R. Cluckers and F. Loeser, "Constructible motivic functions and motivic integration”, Invent. Math. 173:1 (2008), 23-121. MR 2009g:14018 Zbl 1179.14011

[Cluckers and Loeser 2012] R. Cluckers and F. Loeser, "Motivic integration in all residue field characteristics for Henselian discretely valued fields of characteristic zero", preprint, 2012. arXiv 1102.3832

[Demazure and Grothendieck 1970a] M. Demazure and A. Grothendieck (editors), Schémas en groupes, I: Propriétés générales des schémas en groupes, Lecture Notes in Mathematics 151, Springer, Berlin, 1970. MR 43 \#223a Zbl 0207.51401

[Demazure and Grothendieck 1970b] M. Demazure and A. Grothendieck (editors), Schémas en groupes, II: Groupes de type multiplicatif, et structure des schémas en groupes généraux, Lecture Notes in Mathematics 152, Springer, Berlin, 1970. MR 43 \#223b Zbl 0209.24201

[Denef 1984] J. Denef, "The rationality of the Poincaré series associated to the $p$-adic points on a variety”, Invent. Math. 77:1 (1984), 1-23. MR 86c:11043 Zbl 0537.12011 
[Gross and Gan 1999] B. H. Gross and W. T. Gan, "Haar measure and the Artin conductor", Trans. Amer. Math. Soc. 351:4 (1999), 1691-1704. MR 99f:20078 Zbl 0991.20033

[Grothendieck and Dieudonné 1967] A. Grothendieck and J. Dieudonné, "Éléments de géométrie algébrique, IV: Étude locale des schémas et des morphismes de schémas, IV", Inst. Hautes Études Sci. Publ. Math. 32 (1967), 361. MR 39 \#220 Zbl 0153.22301

[Grothendieck et al. 1972] A. Grothendieck, M. Raynaud, and D. S. Rim (editors), Groupes de monodromie en géométrie algébrique, I, Lecture Notes in Mathematics 288, Springer, Berlin, 1972. MR 50 \#7134 Zbl 0237.00013

[Halle and Nicaise 2010] L. H. Halle and J. Nicaise, "The Néron component series of an abelian variety”, Math. Ann. 348:3 (2010), 749-778. MR 2011j:11105 Zbl 1245.11072

[Halle and Nicaise 2011] L. H. Halle and J. Nicaise, "Motivic zeta functions of abelian varieties, and the monodromy conjecture", Adv. Math. 227:1 (2011), 610-653. MR 2012c:14050 Zbl 1230.11076

[Loeser and Sebag 2003] F. Loeser and J. Sebag, "Motivic integration on smooth rigid varieties and invariants of degenerations", Duke Math. J. 119:2 (2003), 315-344. MR 2004g:14026 Zbl 1078.14029

[Nicaise 2009] J. Nicaise, "A trace formula for rigid varieties, and motivic Weil generating series for formal schemes”, Math. Ann. 343:2 (2009), 285-349. MR 2010b:14043 Zbl 1177.14050

[Nicaise 2011] J. Nicaise, "A trace formula for varieties over a discretely valued field", J. Reine Angew. Math. 650 (2011), 193-238. MR 2012d:14039 Zbl 1244.14017

[Nicaise and Sebag 2008] J. Nicaise and J. Sebag, "Motivic Serre invariants and Weil restriction", J. Algebra 319:4 (2008), 1585-1610. MR 2009e:14041 Zbl 1211.14015

[Nicaise and Sebag 2011a] J. Nicaise and J. Sebag, "The Grothendieck ring of varieties", pp. 145-188 in Motivic integration and its interactions with model theory and non-Archimedean geometry, I, edited by R. Cluckers et al., London Math. Soc. Lecture Note Ser. 383, Cambridge Univ. Press, 2011. MR 2885336 Zbl 06059495

[Nicaise and Sebag 2011b] J. Nicaise and J. Sebag, "Motivic invariants of rigid varieties, and applications to complex singularities", pp. 244-304 in Motivic integration and its interactions with model theory and non-Archimedean geometry, I, edited by R. Cluckers et al., London Math. Soc. Lecture Note Ser. 383, Cambridge Univ. Press, 2011. MR 2885338 Zbl 06059497

[Pas 1989] J. Pas, “Uniform p-adic cell decomposition and local zeta functions", J. Reine Angew. Math. 399 (1989), 137-172. MR 91g:11142 Zbl 0666.12014

[Pas 1990] J. Pas, "Cell decomposition and local zeta functions in a tower of unramified extensions of a p-adic field”, Proc. London Math. Soc. (3) 60:1 (1990), 37-67. MR 91g:11143 Zbl 0659.12017

Communicated by Ehud Hrushovski

Received 2011-04-28 Revised 2013-02-01 Accepted 2013-03-03

raf.cluckers@math.univ-lille1.fr

loeser@math.jussieu.fr

johannes.nicaise@wis.kuleuven.be
Laboratoire Painlevé, Université Lille 1, UMR CNRS 8524, Cité Scientifique, 59655 Villeneuve d'Ascq, France http://math.univ-lille1.fr/ cluckers

Institut de Mathématiques de Jussieu, Université Pierre et Marie Curie, UMR CNRS 7586, 4 place Jussieu, 75252 Paris, France http://www.math.jussieu.fr/ loeser

Department of Mathematics, Katholieke Universiteit Leuven, Celestijnenlaan 200B, 3001 Heverlee, Belgium https://perswww.kuleuven.be/ u0025871/ 


\section{Algebra \& Number Theory}

msp.org/ant

\section{EDITORS}

MANAGING EDITOR

Bjorn Poonen

Massachusetts Institute of Technology

Cambridge, USA

\author{
EDITORIAL BOARD CHAIR \\ David Eisenbud \\ University of California \\ Berkeley, USA
}

\section{BOARD OF EDITORS}

Georgia Benkart

Dave Benson

Richard E. Borcherds

John H. Coates

J-L. Colliot-Thélène

Brian D. Conrad

Hélène Esnault

Hubert Flenner

Edward Frenkel

Andrew Granville

Joseph Gubeladze

Ehud Hrushovski

Craig Huneke

Mikhail Kapranov

Yujiro Kawamata

János Kollár

Yuri Manin

Barry Mazur

Philippe Michel
University of Wisconsin, Madison, USA

University of Aberdeen, Scotland

University of California, Berkeley, USA

University of Cambridge, UK

CNRS, Université Paris-Sud, France

University of Michigan, USA

Freie Universität Berlin, Germany

Ruhr-Universität, Germany

University of California, Berkeley, USA

Université de Montréal, Canada

San Francisco State University, USA

Hebrew University, Israel

University of Virginia, USA

Yale University, USA

University of Tokyo, Japan

Princeton University, USA

Northwestern University, USA

Harvard University, USA

École Polytechnique Fédérale de Lausanne
Susan Montgomery

Shigefumi Mori

Raman Parimala

Jonathan Pila

Victor Reiner

Karl Rubin

Peter Sarnak

Joseph H. Silverman

Michael Singer

Vasudevan Srinivas

J. Toby Stafford

Bernd Sturmfels

Richard Taylor

Ravi Vakil

Michel van den Bergh

Marie-France Vignéras

Kei-Ichi Watanabe

Efim Zelmanov
University of Southern California, USA

RIMS, Kyoto University, Japan

Emory University, USA

University of Oxford, UK

University of Minnesota, USA

University of California, Irvine, USA

Princeton University, USA

Brown University, USA

North Carolina State University, USA

Tata Inst. of Fund. Research, India

University of Michigan, USA

University of California, Berkeley, USA

Harvard University, USA

Stanford University, USA

Hasselt University, Belgium

Université Paris VII, France

Nihon University, Japan

University of California, San Diego, USA

\section{PRODUCTION}

production@msp.org

Silvio Levy, Scientific Editor

See inside back cover or msp.org/ant for submission instructions.

The subscription price for 2013 is US \$200/year for the electronic version, and \$350/year ( $\$ 40$, if shipping outside the US) for print and electronic. Subscriptions, requests for back issues and changes of subscribers address should be sent to MSP.

Algebra \& Number Theory (ISSN 1944-7833 electronic, 1937-0652 printed) at Mathematical Sciences Publishers, 798 Evans Hall \#3840, c/o University of California, Berkeley, CA 94720-3840 is published continuously online. Periodical rate postage paid at Berkeley, CA 94704, and additional mailing offices.

ANT peer review and production are managed by EditFLOW ${ }^{\circledR}$ from Mathematical Sciences Publishers.

\section{PUBLISHED BY}

- mathematical sciences publishers

nonprofit scientific publishing

http://msp.org/

(C) 2013 Mathematical Sciences Publishers 


\section{Algebra \& Number Theory}

$\begin{array}{lll}\text { Volume } 7 & \text { No. } 4 \quad 2013\end{array}$

Explicit Chabauty over number fields

SAMIR SIKSEK

Moduli spaces for point modules on naïve blowups

Thomas A. Nevins and Susan J. Sierra

Density of rational points on certain surfaces

Sir Peter SWInNerton-Dyer

HENRIK RUSSELL

Chai's conjecture and Fubini properties of dimensional motivic integration

RAF Cluckers, François LoESER and JohanNes NiCAISE

SHUNSUKE TAKAGI

Finitely presented exponential fields

JONATHAN KIRBY

On a problem of Arnold: The average multiplicative order of a given integer

PÄr Kurlberg and CARL POMERANCE

An analogue of Sturm's theorem for Hilbert modular forms 